\title{
Molecular epidemiology and strain-specific characteristics of Streptococcus agalactiae at the herd and cow level
}

\author{
Y. S. Mahmmod, ${ }^{*} \dagger^{1}$ I. C. Klaas, $\dagger$ J. Katholm, $\ddagger^{2}$ M. Lutton, $\S^{3}$ and R. N. Zadoks $\sharp^{4}$ \\ *Infectious Diseases, Department of Animal Medicine, Faculty of Veterinary Medicine, Zagazig University, Zagazig City 44511, Sharkia Province, \\ Egypt \\ †Department of Large Animal Sciences, Faculty of Health and Medical Sciences, University of Copenhagen, DK-1870 Frederiksberg C, Denmark \\ ¥Knowledge Centre for Agriculture, Cattle, Agro Food Park 15, Skejby, DK-8200 Aarhus N, Denmark \\ $\S M o r e d u n$ Research Institute, Pentlands Science Park, Bush Loan, Penicuik, EH26 OPZ, United Kingdom \\ \#Institute of Biodiversity, Animal Health and Comparative Medicine, College of Medical, Veterinary and Life Sciences, University of Glasgow, \\ Glasgow G61 1QH, United Kingdom
}

\section{ABSTRACT}

Host-adaptation of Streptococcus agalactiae subpopulations has been described whereby strains that are commonly associated with asymptomatic carriage or disease in people differ phenotypically and genotypically from those causing mastitis in dairy cattle. Based on multilocus sequence typing (MLST), the most common strains in dairy herds in Denmark belong to sequence types (ST) that are also frequently found in people. The aim of this study was to describe epidemiological and diagnostic characteristics of such strains in relation to bovine mastitis. Among 1,199 cattle from 6 herds, cowlevel prevalence of $S$. agalactiae was estimated to be $27.4 \%$ based on PCR and $7.8 \%$ based on bacteriological culture. Quarter-level prevalence was estimated at 2.8\% based on bacteriological culture. Per herd, between 2 and 26 isolates were characterized by pulsed-field gel electrophoresis (PFGE) and MLST. Within each herd, a single PFGE type and ST predominated, consistent with a contagious mode of transmission or point source infection within herds. Evidence of within-herd evolution of $S$. agalactiae was detected with both typing methods, although ST belonged to a single clonal complex (CC) per herd. Detection of CC23 (3 herds) was associated with significantly lower approximate count (colony-forming units) at the quarter level and significantly lower cycle threshold value at the cow level than detection of CC1 (2 herds) or CC19 (1 herd), indicating a lower bacterial load in CC23 infections. Median

Received January 29, 2015.

Accepted June 9, 2015.

${ }^{1}$ Current address: Department of Veterinary Population Medicine, University of Minnesota, Minneapolis, MN.

${ }^{2}$ Current address: DNA Diagnostic A/S, Voldbjergvej 16, 8240 Risskov, Denmark.

${ }^{3}$ Current address: Liverpool School of Tropical Medicine, Pembroke Place, Liverpool, L3 5QA, United Kingdom.

${ }^{4}$ Corresponding author: ruth.zadoks@moredun.ac.uk values for the number of infected quarters and somatic cell count (SCC) were numerically but not significantly lower for cows infected with $\mathrm{CC} 23$ than for cows with CC1 or CC19. For all CC, an SCC threshold of 200,000 cells/mL was an unreliable indicator of infection status, and prescreening of animals based on SCC as part of $S$. agalactiae detection and eradication campaigns should be discouraged.

Key words: Streptococcus agalactiae, molecular epidemiology, multilocus sequence typing, pulsed-field gel electrophoresis

\section{INTRODUCTION}

Streptococcus agalactiae was the predominant cause of bovine mastitis in Europe in the 1950s and 1960s (Stableforth, 1950; Andersen et al., 2003) when evidence-based mastitis control programs were developed. Programs based on reductions in both the duration and incidence of new infections were very effective in reducing the prevalence of $S$. agalactiae mastitis but less effective in reducing the prevalence of other streptococcal species (Neave et al., 1969; Jensen, 1980a). In several countries, $S$. agalactiae is now extremely rare in dairy cattle. For example, 92 positive cases were detected among 178,688 quarter milk samples in Belgium, no $S$. agalactiae was detected in 4,220 quarter milk samples from the Netherlands, and no pure culture of $S$. agalactiae was detected in 85,576 quarter milk samples from Canada, suggesting a quarter-level prevalence of $0.05 \%$ or less in those countries (Piepers et al., 2007; Sampimon et al., 2009; Reyher et al., 2011). In other European countries, S. agalactiae is detected more frequently; for example, in $0.7 \%$ of 10,000 quarter milk samples from a survey in Germany, with positive cows found on a large proportion of farms (29\% of 80 farms; Tenhagen et al., 2006). Prevalence estimates that are based on submissions to diagnostic laboratories rather 
than on prospective surveys show higher values; for example, $2.3 \%$ of 18,711 composite or quarter milk samples in Sicily and $6.5 \%$ of 8,204 samples in Estonia were positive for S. agalactiae (Ferguson et al., 2007; Kalmus et al., 2011).

In some countries, successful control of $S$. agalactiae has been followed by its re-emergence. In Denmark, mandatory annual surveillance of dairy herds for presence of $S$. agalactiae in bulk tank milk (BTM) is in place, with registration of infected herds and, until 2005 , restrictions on the sale of livestock from such herds. Despite these control measures, the prevalence increased from approximately $2 \%$ in 2000 to $6.1 \%$ in 2009 based on bacteriological culture (BC) of BTM samples (Katholm et al., 2012).

The success of traditional mastitis control programs was largely because $S$. agalactiae was primarily spread from cow to cow. For this transmission pattern, a reduction in prevalence contributes to reductions in incidence, creating a positive feedback loop for control. Cow-to-cow transmission results in the presence of a single $S$. agalactiae strain per herd, as observed repeatedly through use of comparative strain typing methods (Zadoks et al., 2011). Although comparative typing is very useful for within-herd analyses, definitive typing methods such as multilocus sequence typing (MLST) are preferable for comparisons on a large scale; for example, across herds or host species (Struelens et al., 1998; Enright and Spratt, 1999). Strain typing of bovine BTM isolates of $S$. agalactiae from Denmark using MLST showed that the most frequent sequence types (ST) were ST1, ST23, and ST103 (Zadoks et al., 2011). Carriage of ST1 or ST23 is common in the urogenital and gastrointestinal tract of healthy men and women (Persson et al., 1986; Bliss et al., 2002; Manning et al., 2009) and it has been suggested that humans may occasionally be a source of infection for cows (Jensen, 1980b; Dogan et al., 2005).

Jensen (1982) used human and bovine S. agalactiae isolates to induce mastitis experimentally and observed strain-related differences in clinical manifestation, duration of IMI, and bacterial shedding by infected cows. Shorter duration of IMI and lower bacterial shedding were linked to a lower tendency to spread within herds and Jensen suggested that a program to eradicate $S$. agalactiae should focus on the control of bovine strains because human strains tended to disappear spontaneously (Jensen, 1980b, 1982). It is unknown where the strains described by Jensen (1980b, 1982) would fit in an MLST-based population snapshot of human and bovine $S$. agalactiae. Despite the preponderance of ST1 and ST23 in Danish BTM and the potential human origin of those strains, most $S$. agalactiae IMI in Danish cattle are chronic and subclinical, which does not match Jensen's descriptions of bovine IMI with humanderived material.

For development of diagnostic and control programs to combat the re-emergence of $S$. agalactiae mastitis, it is important to understand (a) the mode of transmission of the most common $S$. agalactiae strains in modern herds, and (b) whether they differ from each other in commonly used diagnostic characteristics such as SCC, culture results (number of colony-forming units; cfu) or real-time PCR cycle threshold $(\mathbf{C t})$ values, all of which affect our ability to detect infections. In this study, we examined within-herd epidemiology and diagnostic characteristics of IMI in herds with potentially human-derived ST of S. agalactiae.

\section{MATERIALS AND METHODS}

\section{Herd Selection}

Data and isolates were obtained from 6 dairy herds in Jutland, Denmark, that participated in a project on associations between BTM PCR results and the prevalence of S. agalactiae (Mahmmod et al., 2013). To confirm that $S$. agalactiae was present in the selected herds, monthly PCR BTM tests were conducted from January to March 2011, and Ct values $<37$ were obtained in at least 2 of 3 tests. Routine milking practices in the herds included cleaning of teats with individual boiled towels (5 herds) or excelsior wood wool (1 herd), and use of postmilking teat dipping (5 herds). Premilking teat dipping was not practiced in any herd. Cows in all herds were mainly Danish Holstein-Friesians. Herd size ranged from 146 to 372 animals, average cow-level SCC at sampling ranged from $249 \times 10^{3}$ cells $/ \mathrm{mL}$ to 381 $\times 10^{3}$ cells $/ \mathrm{mL}$, and average ECM production ranged from 23.0 to $32.8 \mathrm{~kg} / \mathrm{d}$ (Table 1 ). We regarded these 6 herds as representative of modern Danish dairy herds with $S$. agalactiae.

\section{Cow Selection and Sampling}

Between March 28 and May 28, 2011, each herd was visited by 2 trained technicians from the Knowledge Centre for Agriculture, Cattle (Aarhus, Denmark) with visits coordinated to coincide with routine DHI sampling. After farm personnel had carried out their routine premilking practices, quarter milk samples for bacteriological examination were collected from approximately $50 \%$ of cows before attachment of milking units. Inclusion of cows was based on systematic random sampling; that is, in herringbone or side-by-side parlors, cows milked with the first, third, and fifth units, and so on, on one side of the parlor were included, as well as cows milked with the second, fourth, and sixth 
unit, and so on, on the other side. In the herd with a rotary parlor, cows milked with every second milking unit were included. Samples $(\sim 5 \mathrm{~mL})$ were collected aseptically in sterile snap-cap plastic tubes following standard procedures (National Mastitis Council, 1999) and placed in ice boxes with an internal temperature of approximately $5^{\circ} \mathrm{C}$. Within $12 \mathrm{~h}$ of sample collection, ice boxes were delivered to the Eurofins Steins Laboratory (Holstebro, Denmark), where they were used for BC. Composite milk samples for PCR testing were taken automatically, but not aseptically, from all cows, using routine procedures for collection of DHI samples. During the milking of each cow, the Tru-Test Electronic Milk Meter device (Tru-Test Group Company, New Zealand) automatically took predefined portions of milk and dispensed a representative composite sample into the barcoded sample vial when milking of the cow had finished. The bronopol preserved milk samples were delivered to Eurofins Steins Laboratory within $24 \mathrm{~h}$ of collection and used for real-time PCR.

\section{Diagnostic Testing}

For each milk sample, aliquots of $0.01 \mathrm{~mL}$, as measured using calibrated plastic loops, were streaked aseptically onto calf blood agar (Statens Serum Institut, Copenhagen, Denmark), CHROMagar StrepB (Th. Geyer Denmark ApS, Roskilde, Denmark) and S. agalactiae selective medium (N-plate medium; Mahmmod et al., 2013). Plates were incubated aerobically at $37^{\circ} \mathrm{C}$ for 18 to $24 \mathrm{~h}$ and examined for growth. No samples were excluded as contaminated based on the definition of isolation of 3 or more types of colonies (National Mastitis Council, 1999). Species identity of suspected $S$. agalactiae colonies was phenotypically confirmed using the Christie, Atkins, Munch-Petersen (CAMP) test (National Mastitis Council, 1999). If a cow had at least one quarter positive for $S$. agalactiae, the cow was considered test-positive for $\mathrm{BC}$ and the number of positive quarters was recorded. The counts (cfu) of $S$. agalactiae identified on selective medium were recorded at the quarter level. Because the inoculum size was standardized but relatively small, we considered these to be approximate counts (Acfu). One representative colony per quarter was transferred to new blood agar plates to obtain pure culture for subsequent archiving and molecular characterization.

Composite milk samples were tested for presence of $S$. agalactiae DNA using the PathoProof Mastitis Complete-12 kit (Thermo Fisher Scientific, Vantaa, Finland). The Ct values were recorded, with lower $\mathrm{Ct}$ values indicative of higher DNA concentrations (Koskinen et al., 2009). Samples were considered positive at $\mathrm{Ct}$ value $\leq 37$ in accordance with guidelines for indi- 
vidual samples, which differ from guidelines for BTM samples (Koskinen et al., 2009; Katholm et al., 2012). Somatic cell count was estimated from the routinely collected samples at Eurofins Steins Laboratory by a fluoro-optoelectronic method using Fossomatic measurements (Foss-Electric A/C, Hillerød, Denmark).

\section{Molecular Characterization}

Pure cultures were used to inoculate brain heart infusion broth (BHI; Oxoid, Basingstoke, UK) which was incubated aerobically overnight at $37^{\circ} \mathrm{C}$ without shaking. Overnight cultures were used to prepare bacterial lysates using lysozyme and proteinase $\mathrm{K}$. The species identity of all lysates was confirmed by $S$. agalactiae-specific PCR reaction using primers STRA-AgI (5'-AAGGAAACCTGCCATTTG-3') and STRA-AgII (5'-TTAACCTAGTTTCTTTAAAACTAGAA-3'), which target the 16S-23S intergenic spacer region (Phuektes et al., 2001). Broth cultures were also used to prepare plugs for pulsed-field gel electrophoresis (PFGE) with restriction enzyme SmaI, as detailed elsewhere (Delannoy et al., 2013). When running the PFGE gels, isolates were grouped by herd to allow for direct comparison of patterns between quarters and cows within herds. In addition, multiple lanes of a control strain (STIR-CD-25) were included on each gel to allow for comparison of patterns across herds and gels. This control strain, which was originally isolated from a diseased tilapia and belongs to ST283, has a good distribution of bands across the expected range of the PFGE band sizes (Delannoy et al., 2013). Banding patterns were interpreted by 2 independent observers using criteria defined by Tenover and colleagues (1995). When patterns within a herd differed by up to 3 bands representing a single genetic event, they were considered epidemiologically related and identified as a main type, indicated by a capital letter, and a subtype, indicated by a numerical suffix. When patterns within a herd differed by 4 to 6 bands, they were identified as possibly related (Tenover et al., 1995). In addition, computer-assisted comparison of banding patterns was used to assess reproducibility of marker strain patterns across gels and to create dendrograms. This was done using Phoretix 1D Pro with PFGE clusters identified at $75 \%$ similarity (TotalLab, Newcastle upon Tyne, UK). Finally, MLST was performed for all isolates using conventional PCR, gel electrophoresis, and sequence analysis of purified DNA fragments (Jones et al., 2003) or by HiMLST, which is a commercial high-throughput MLST service (provided by Streeklab Haarlem, Haarlem, the Netherlands) targeting the same loci as conventional MLST (Boers et al., 2012). Sequence types were assigned to clusters of closely related ST or clonal complexes (CC) using Springman et al.'s (2014) modification of the definition originally proposed by Feil et al. (2004).

\section{Statistical Analysis}

Data on parity, SCC, and DIM were extracted from the Danish cattle database and merged with the laboratory results for BC, Acfu, Ct values, and molecular typing data at the CC level. Somatic cell count and Acfu count were normalized by natural log-transformation (lnSCC and $\ln \mathrm{Acfu}$, respectively) whereas Ct value was normally distributed and not transformed. Generalized linear models were specified in the MIXED procedure of SAS (SAS 9.3, SAS Institute Inc., Cary, NC) using $\operatorname{lnSCC}$, Ct value, or lnAcfu as outcome variable, and CC, parity, number of infected quarters, and DIM as independent variables. Clonal complex was included as categorical variable (3 levels: CC1, CC19, or CC23), parity was included as continuous variable, and number of infected quarters was included as categorical variable (2 levels: 1 quarter or $>1$ quarter). For DIM, categories were defined based on visualization of the results of a generalized additive model (gam procedure), which indicated a nonlinear relationship with 2 turning points (150 DIM and 280 DIM) between DIM and the outcome variables. Variables that were not significant based on type 3 tests of fixed effects were removed using a backward elimination procedure, with the exception of CC, which was kept in the model because it was the main variable of interest. For the 3 categorical variables, the last level (CC23, >1 quarter, and >280 DIM, respectively) was used as the reference level. For variables with more than 2 levels (i.e., CC and DIM), the significance of differences between levels was assessed using differences between least squares means. Herd (H1 to H6) was initially included as a random factor to account for within-herd clustering in all 3 models but herd effect was not significant and it was omitted from the final models. Because of the nature of the data, models for $\operatorname{lnSCC}$ and $\mathrm{Ct}$ value were run at the cow level, whereas the model for $\ln A c f u$ was run at the quarter level. For 3 animals in H4, PCR data were not available. Those animals were excluded from analysis of $\mathrm{Ct}$ values. When cow was included in the quarter-level model, it would not converge due to the large proportion of cows with a single infected quarter. Therefore, no correction for clustering was used. The association between CC and number of infected quarters $(1$ or $>1)$ was tested by means of Fisher's exact test. To reduce the probability of type II errors, significance for statistical analyses was declared at $P<0.10$. 


\section{RESULTS AND DISCUSSION}

\section{Prevalence at Species and Strain Level}

Cow-level prevalence of $S$. agalactiae within herds was $27.4 \%$ (range 8.3 to $66.7 \%$ ) based on PCR and $7.8 \%$ (range 1.6 to $21.7 \%$ ) based on $\mathrm{BC}$, whereas quarter-level prevalence, which was calculated based on BC, was $2.8 \%$ (range 0.4 to $7.8 \%$; Table 1 ). Polymerase chain reaction is known to be significantly more sensitive than BC for detection of $S$. agalactiae in composite cow milk samples (Mahmmod et al., 2013). When collecting milk samples automatically for PCR, as was done in the current study, there is a risk of carryover, which may lead to slight overestimation of $S$. agalactiae prevalence (Mahmmod et al., 2014). Moreover, PCR does not provide information about the number of infected quarters. Based on BC, the proportion of cows with multiple infected quarters ( 15 of $49,31 \%$; Table 1) was much higher than expected under the assumption of independence of quarters within cows, which is in agreement with previous publications (Barkema et al., 1997). A threshold of 200,000 cells $/ \mathrm{mL}$ is often used to differentiate infected cows from noninfected cows (Schukken et al., 2003). In most herds (5 of 6 ), at least one $S$. agalactiae culture-positive animal had a composite SCC $<200,000$ cells $/ \mathrm{mL}$ at routine DHI testing, and the proportion of cows with SCC $<200,000$ cells $/ \mathrm{mL}$ was $21 \%$ across the study. Similarly, in an evaluation of the Danish S. agalactiae eradication program covering the years 1989 to 2002, 25.8\% of 8,268 S. agalactiae culture-positive quarters had a California Mastitis Test value of 1 (Runge et al., 2004). The combination of pathogen detection with normal SCC has been described as "latent infection." In one study of S. agalactiae mastitis, where SCC $<500,000$ cells $/ \mathrm{mL}$ was used as cut-off, $50 \%$ of BC-positive animals were considered to have latent infections (Thieme and Haasmann, 1978). By that measure, the majority of BCpositive cows $(30 / 49)$ in our study would have latent infection. Regardless of the choice of SCC threshold value, prescreening of cows based on SCC before sampling for BC or PCR cannot be recommended as part of $S$. agalactiae eradication efforts.

Data from PFGE and MLST were generated for $S$. agalactiae isolates from 71 quarters of 49 BC-positive cows. Pulsed-field gel electrophoresis requires availability of viable bacteria because amplification of the organism's genetic material before enzymatic digestion occurs during bacterial growth. In contrast, PCR or sequence-based methods can be applied to DNA extracts from nonviable organisms. Attempts to conduct MLST directly on DNA extracts from milk, however, were not successful (data not shown), and culture of isolates was needed to obtain DNA extracts of sufficient quality for MLST. Hence, all strain-typing methods in this study required $\mathrm{BC}$ as a first step and it is unknown whether PCR-positive, culture-negative quarters shed the same strains as PCR-positive, culture-positive quarters.

As in previous studies (reviewed in Zadoks et al., 2011), each herd had a dominant and herd-specific PFGE type (Table 2, Figure 1), consistent with a contagious mode of transmission within herds, or exposure to a point source of infection. In each herd, a dominant ST was identified (Table 2, Figure 1), as previously reported from a herd in India (Shome et al., 2012). The dominant ST could be the same across multiple herds, in agreement with results from annual BTM surveillance in Denmark, which showed that ST1 and ST23 were the most prevalent ST at the herd level (Zadoks et al., 2011). Both types, as well as ST19, which was the dominant type in one herd, have also been identified in asymptomatic human carriers and patients. In humans, ST1 has been isolated significantly more frequently from asymptomatic carriers than from patients, whereas the reverse is true for ST19 (Manning et al., 2009); ST19 has been reported from cattle before but is less prevalent among bovine isolates than ST1 or ST23 (Sørensen et al., 2010; Zadoks et al., 2011; Springman et al., 2014).

\section{Within-Herd Molecular Epidemiology}

Typing results were uniform within 2 herds (H1 and $\mathrm{H} 5)$. In the other herds, within-herd heterogeneity in typing results was observed. In H3, heterogeneity was only observed in MLST results whereas in H4, heterogeneity was only observed in PFGE results (Figure 1, Table 2). Differentiation by PFGE within ST and, conversely, differentiation by MLST within PFGE types is known to occur in S. agalactiae (Shome et al., 2012; Delannoy et al., 2013; Yang et al., 2013). In the remaining 2 herds, heterogeneity was found in both PFGE and MLST results. Of 3 cows in $\mathrm{H} 2,1$ cow tested positive for the main PFGE type and ST, 1 for a PFGE subtype and the main ST, and 1 for the main PFGE type and an MLST subtype; that is, a single locus variant (SLV; Table 2). Finally, in H6, 24 of 25 isolates belonged to ST19 and 1 isolate belonged to ST44, which is an SLV of ST19. Isolates from H6 were more heterogeneous by PFGE than by MLST, and 4 clusters were identified at $75 \%$ banding pattern similarity (Figure 1). Eight isolates belonged to the main PFGE type and 8 isolates belonged to 1 of 5 subtypes that differed from the main type by at most 3 bands, indicating probable relatedness. The PFGE patterns for the remaining 9 isolates differed from the main PFGE type by 4 to 6 bands, 
Table 2. Overview of molecular typing results showing the number of quarters and cows per herd infected with Streptococcus agalactiae of the specified pulsed-field gel electrophoresis (PFGE) type or sequence type (ST) and clonal complex (CC)

\begin{tabular}{lcclrr}
\hline Herd & Cows $^{1}$ & Quarters & PFGE type & ST & CC $^{3}$ \\
\hline 1 & 7 & 8 & H1-A & 23 & 23 \\
2 & 1 & 2 & H2-A & 1 & 1 \\
& 1 & 2 & H2-A.1 & 1 & 1 \\
3 & 1 & 1 & H2-A & 458 & 1 \\
& 1 & 1 & H3-A & 199 & 23 \\
4 & 1 & 1 & H3-A & 1 & 1 \\
& 14 & 20 & H4-A & 1 & 1 \\
5 & 4 & 5 & H4-B & 1 & 1 \\
6 & 1 & 1 & H4-C & 19 & 1 \\
& 3 & 5 & H5-A & 19 & 19 \\
& 7 & 7 & H6-A & 19 & 19 \\
& 7 & 9 & H6-A.2 to A.5 & 44 & 19 \\
\hline
\end{tabular}

${ }^{1}$ Cows that were infected with different strains in different quarters were counted multiple times. As a result, the total for this column exceeds the number of cows. The column total for quarters is equal to the actual number of $S$. agalactiae-positive quarters.

${ }^{2} \mathrm{H}$ refers to herd number, capital letters (A, B, C) refer to main PFGE types within herds, numeral suffixes (.1 to .5) refer to subtypes within main PFGE types (1 to 3 band differences, classified as probably related based on criteria defined by Tenover et al., 1995).

${ }^{3} \mathrm{CC}$ nomenclature is based on Springman et al.'s (2014) modification of the original definition by Feil et al. (2004).

${ }^{4}$ This cow was infected with PFGE type H4-B in one quarter and H4-C in a different quarter.

${ }^{5}$ Based on 4 to 6 band differences relative to main type, classified as possibly related as per the criteria defined by Tenover et al. (1995).

${ }^{6}$ This cow was infected in 3 quarters, with different PFGE types for each quarter, ST19 in 2 quarters and ST44 in 1 quarter.

indicating possible relatedness (Table 2; Tenover et al., 1995). When multiple quarters of a single cow were characterized, PFGE and MLST results could be consistent across quarters (e.g., Figure 1, H2-C2, H4-C6, and $\mathrm{H} 6-\mathrm{C} 15$, where $\mathrm{H}$ identifies the herd and $\mathrm{C}$ identifies the cow) or different based on PFGE or MLST (e.g., Figure 1, H6-C9 and H6-C16).

The PFGE results from $\mathrm{H} 6$ were unusual, in that the patterns were so diverse that they might not have been recognized as related had not a large number of isolates been typed, which led to identification of both "probably related" and "possibly related" types. In addition, the differences within H6 could be compared to the differences between $\mathrm{H} 6$ and the remaining herds, and within-herd differences were clearly smaller than between-herd differences (Figure 1). The accumulation of mutations in $\mathrm{H} 6$ may reflect the large herd size (n $=372)$, the high proportion of positive animals $(23.3 \%$ based on PCR), and the duration of herd-level infection, which was more than $10 \mathrm{yr}$ based on records from the Danish $S$. agalactiae surveillance program. The size of the infected host population and the temporal scale of an infection are proxy measures for the number of replication cycles of the organism (Struelens et al., 1998). As the organism replicates, mutations accumulate and so do changes in banding patterns (Tenover et al., 1995), which may explain our observations in H6. We cannot rule out the possibility that new strains of S. agalactiae were introduced into $\mathrm{H} 6$ but the clustering of PFGE patterns by herd (Figure 1) suggests that such introductions have not occurred or that newly introduced strains have not persisted in the herd.

The $3 \mathrm{SLV}$ of major ST that were detected in this study (ST458 as SLV of ST1; ST44 as SLV of ST19; and ST199 as SLV of ST23; Table 2) appeared to be due to recombination rather than accumulation of point mutations because the variant allele in each of the SLV differed from the allele in the main ST by at least 5 SNP (http://pubmlst.org/sagalactiae/; last accessed October 20, 2014). Recombination is common in $S$. agalactiae, both in virulence genes and in housekeeping genes (Sørensen et al., 2010; Richards et al., 2011). The occurrence of recombination events within herds raises the question of whether other, undetected strains of $S$. agalactiae may have been present in the herds, possibly in people handling the animals. In contrast to statements in the veterinary literature (Keefe, 1997), $S$. agalactiae is not an obligate intramammary pathogen. Rather, it is a common commensal of people, and between 20 and $50 \%$ of healthy adults carry the organism in their urogenital or gastrointestinal tract (Hansen et al., 2004; Manning et al., 2009). In addition, humans 


\section{Dendrogram: UPGMA(Dice)}

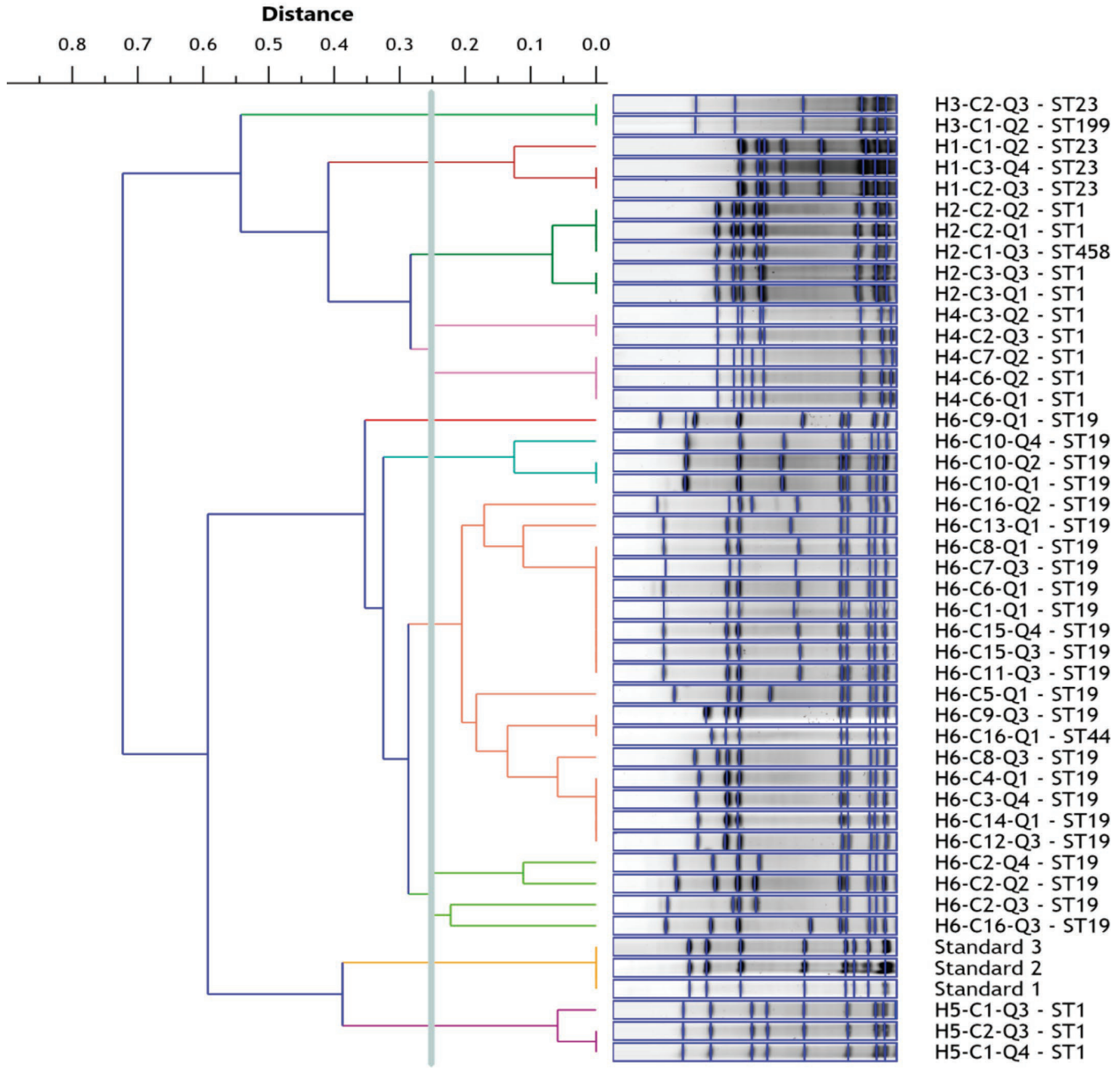

Figure 1. Dendrogram showing examples of pulsed-field gel electrophoresis (PFGE) patterns for Streptococcus agalactiae isolates from quarter milk samples from 6 dairy herds in Denmark. Herd $(\mathrm{H})$, cow $(\mathrm{C})$, and quarter $(\mathrm{Q})$ of origin are shown together with the sequence type (ST) of each isolate. The PFGE clusters were defined by $75 \%$ banding pattern similarity. UPGMA = unweighted pair group method with arithmetic mean. Color version available online.

may carry S. agalactiae in their throat or on their skin (Van der Mee-Marquet et al., 2008), and cattle and sheep may excrete $S$. agalactiae in feces (Manning et al., 2010). Sequence types 1, 19, and 23 are among the most common carriage strains in humans (Van der Mee-Marquet et al., 2008; Manning et al., 2009), providing a likely source of exposure for cattle. Despite the occurrence of occasional SLV within herds, a single isolate from BTM can generally be used to represent the herd in national-scale molecular surveillance. 


\section{Epidemiological and Diagnostic Characteristics at Strain Level}

For analysis of the association of strains with epidemiological and diagnostic characteristics, definitive typing data were used; that is, MLST data, because they allow for comparison across herds, studies, and host species (Enright and Spratt, 1999). To maximize the power of the analysis, MLST data were aggregated at the CC level so that SLV of the main types did not need to be excluded. When the definition for CC was originally proposed by Feil and colleagues (2004), CC1, CC19, and numerous other CC were distinct from each other. Based on the CC definition sensu stricto, these $\mathrm{CC}$ have since been amalgamated into a single $\mathrm{CC}$ that also includes the human neonatal $\mathrm{CC} 17$ and the bovine-associated CC67. To keep the CC nomenclature meaningful, a modified CC definition has been proposed (Springman et al., 2014), which allows for continued use of the descriptors CC1, CC17, CC19, and so on. In our study, using nomenclature as proposed by Springman et al. (2014), data were available for CC1, CC19, and $\mathrm{CC} 23$, with 3,1 , and 2 herds per CC, respectively (Table 3). Due to the predominance of a single CC per herd, CC and herd could not be included in the models simultaneously. Because CC is the variable of specific interest in this study, herd was omitted. This implies that observed strain effects might, in fact, be herd effects, although the inclusion of multiple herds for CC1 and CC23 suggests that the effects, particularly at the cow level, could be at least partly determined by the pathogen and not just by the environment.

Within-herd prevalence of the CC at cow level ranged from 3.1 to $21.7 \%$ for $\mathrm{CC} 1,9.2 \%$ for $\mathrm{CC} 19$, and 3.1 to
$8.8 \%$ for $\mathrm{CC} 23$. When multiple strains of a pathogen coexist within a herd, within-herd prevalence can be seen as a proxy for transmissibility. For several mastitis pathogens; for example, Staphylococcus aureus and Streptococcus uberis, transmissibility has been shown to be strain-specific based on within-herd comparisons (Smith et al., 1998; Zadoks et al., 2003). Because transmission of mastitis pathogens is strongly influenced by herd management, and because only a single strain (main PFGE type, main ST) of $S$. agalactiae was observed in each herd, it was not possible to identify strain-specific transmission in the current study.

The ratio of PCR-based prevalence to BC-based prevalence at the cow level was evaluated because some strains may grow better than others under laboratory conditions. For example, $S$. agalactiae strains belonging to CC552 do not grow on standard blood agar plates at $37^{\circ} \mathrm{C}$ (Delannoy et al., 2013). Differences in growth could lead to strain-specific underestimation of prevalence based on $\mathrm{BC}$, whereas $\mathrm{PCR}$ results would not be affected, resulting in an increased PCR-based prevalence relative to $\mathrm{BC}$-based prevalence. The prevalence ratio based on PCR and BC was similar between CC, with a ratio of 2.3 to 14.8 for CC1 (3 herds), 2.5 for CC19 (1 herd), and 2.3 to 11.7 for CC23 (2 herds).

At the cow level, the association between $\mathrm{CC}$ and number of infected quarters, composite SCC, and $\mathrm{Ct}$ value was evaluated. The descriptive statistics (Figure 2 , top left) indicated that the proportion of cows with 2 or 3 infected quarters was approximately equal for CC1 and CC19 (close to $40 \%$ of positive cows) but lower for CC23 (10\% of positive cows had 2 infected quarters, no animals had more than 2 infected quarters). The observed difference in number of infected

Table 3. Relationship between clonal complex (CC) of Streptococcus agalactiae, cow characteristics, and diagnostic characteristics for 45 culturepositive dairy cows from 6 Danish dairy herds ${ }^{1}$

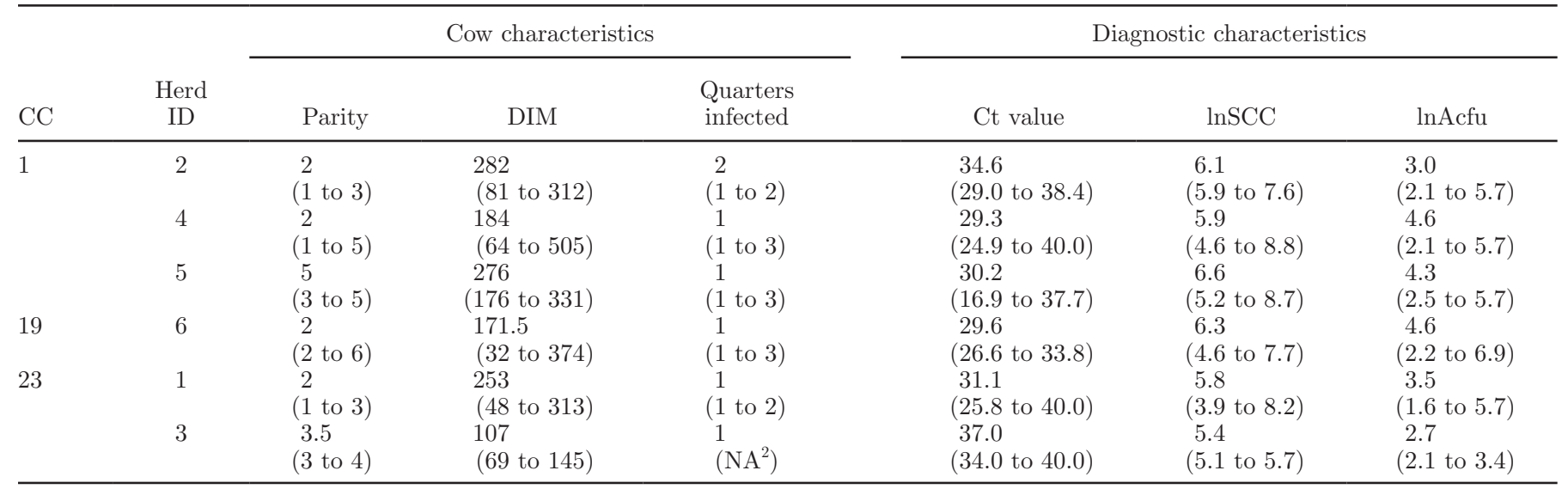

${ }^{1}$ Results are shown as median with range in parentheses. Cycle threshold (Ct value) and log-transformed SCC (lnSCC) are shown at cow level. Log-transformed approximate count (lnAcfu) is shown at quarter level ( $\mathrm{n}=71)$.

${ }^{2}$ Not applicable; that is, all values were the same and there is no range to report. 


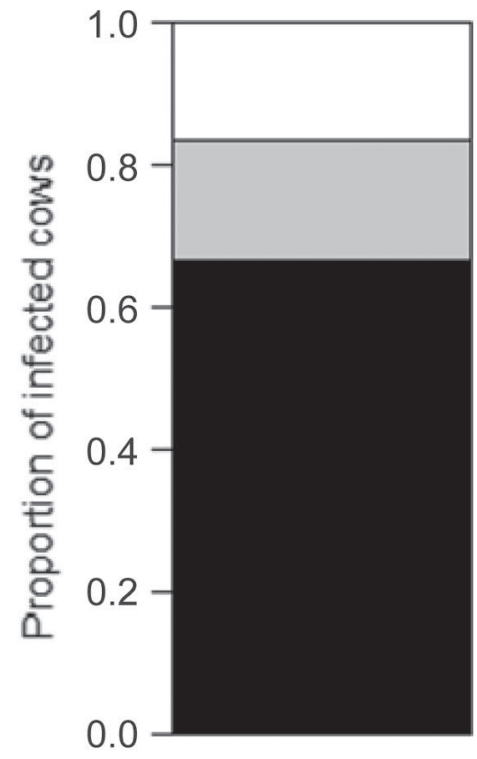

1

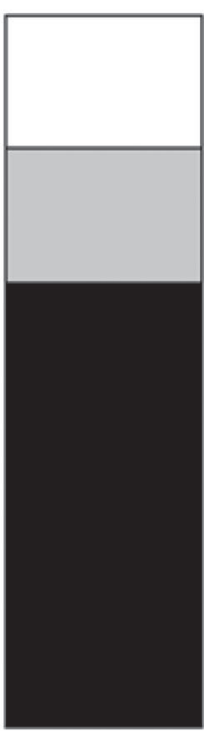

19

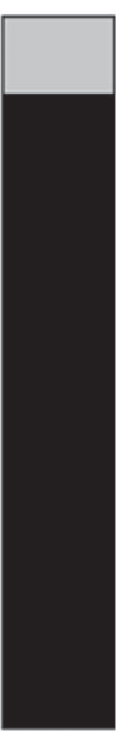

23

$\mathrm{CC}$

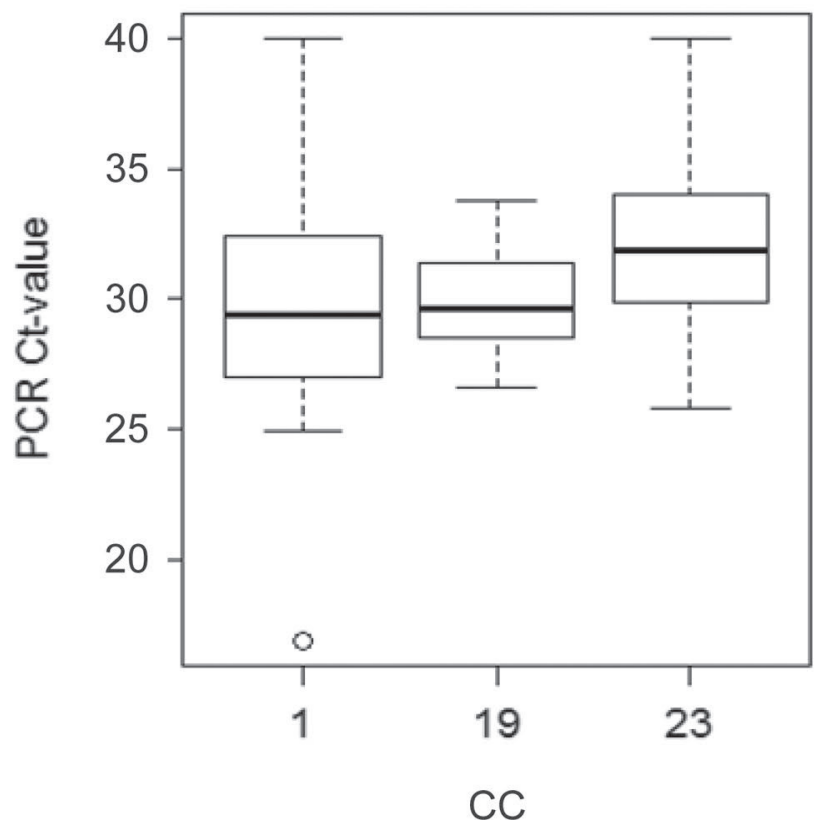

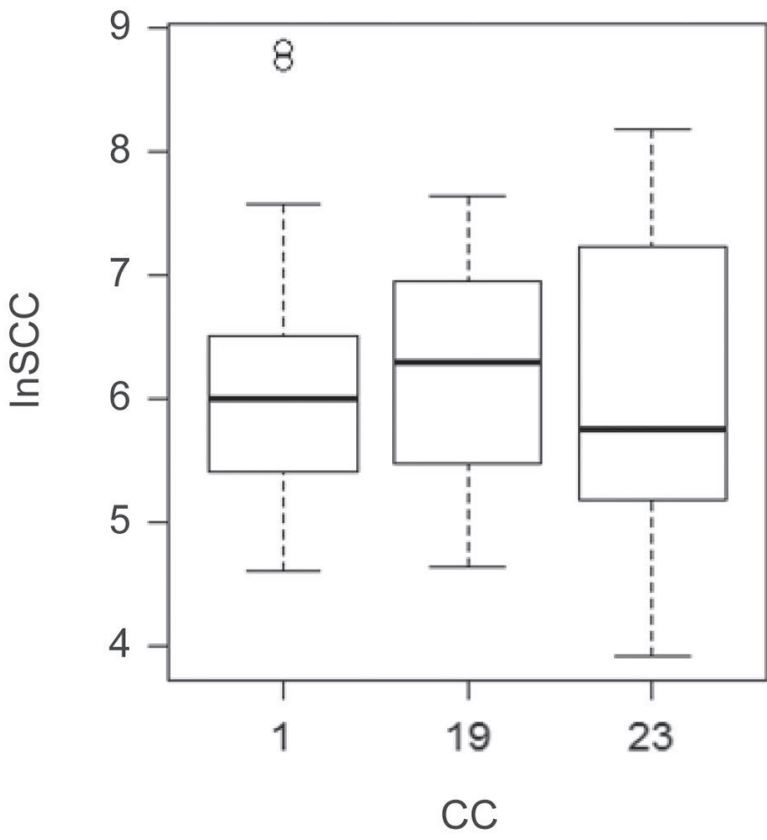

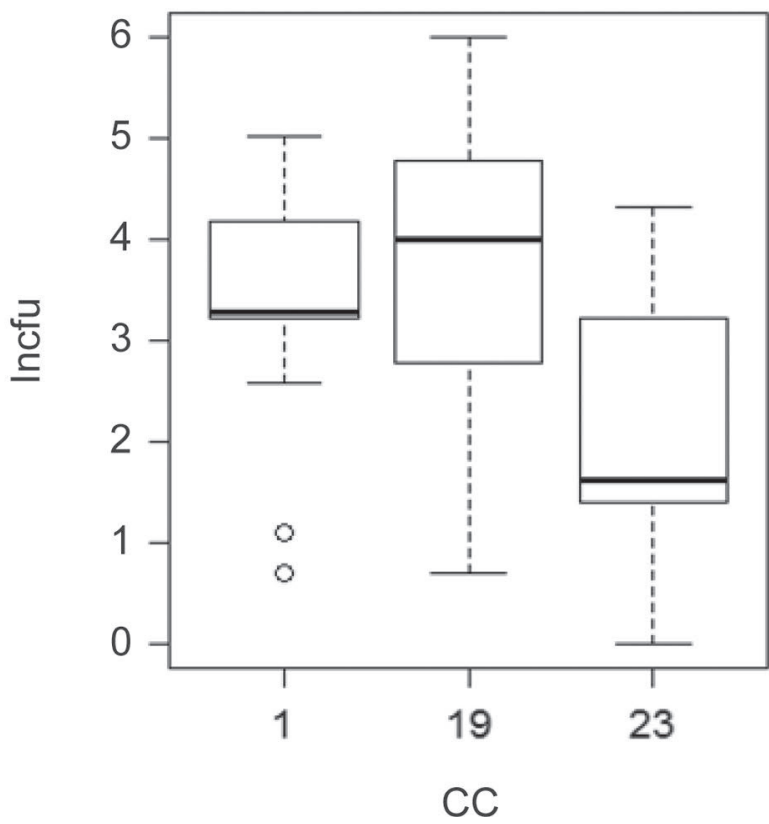

Figure 2. Relationship between clonal complexes of Streptococcus agalactiae and the number of infected quarters per animal (top left; black $=1$ infected quarter; gray $=2$ infected quarters; white $=3$ infected quarters; 49 cows), lnSCC at cow level (top right; 49 cows), PCR cycle threshold (Ct) value at cow level (bottom left; 46 cows), and approximate count (lncfu) at quarter level (bottom right; 71 quarters from 49 cows).

quarters was not significant in the Fisher's exact test. Similarly, descriptive statistics for SCC showed a lower median SCC for cows infected with CC23 than for cows infected with $\mathrm{CC} 1$ or CC19 (Figure 2, top right), but this difference was not significant (Table 4). Somatic cell count increased with parity and with number of 
Table 4. Estimated coefficients of factors potentially associated with cow-level lnSCC in 49 Streptococcus agalactiae-infected cows from 6 herds

\begin{tabular}{|c|c|c|c|c|}
\hline \multirow[b]{2}{*}{ Effect } & \multirow[b]{2}{*}{$\begin{array}{c}\text { Type } 3 \text { test of } \\
\text { fixed effect }(\operatorname{Pr}>\mathrm{F})\end{array}$} & \multicolumn{3}{|c|}{ Solution for fixed effects } \\
\hline & & Estimate & $\mathrm{SE}$ & $\begin{array}{c}P \text {-value } \\
(\operatorname{Pr}>|\mathrm{t}|)\end{array}$ \\
\hline Intercept & - & 5.81 & 0.50 & $<0.001$ \\
\hline Parity & 0.003 & 0.38 & 0.12 & 0.003 \\
\hline Number of infected quarters & 0.018 & - & - & - \\
\hline 1 & - & -0.75 & 0.31 & 0.018 \\
\hline$>1$ & - & Reference & - & - \\
\hline Clonal complex (CC) & 0.93 & - & - & - \\
\hline CC 1 & - & -0.12 & 0.39 & 0.76 \\
\hline CC 19 & - & -0.16 & 0.41 & 0.71 \\
\hline CC 23 & - & Reference & - & - \\
\hline
\end{tabular}

infected quarters. Cows with CC23 had higher median $\mathrm{Ct}$ values than cows with $\mathrm{CC} 1$ or $\mathrm{CC} 19$ and this difference was statistically significant (Figure 2, bottom left; Table 5). The difference in $\mathrm{Ct}$ value shows that bacterial shedding by CC23-infected animals is lower than that by animals infected with CC1 or CC19. The $\mathrm{Ct}$ values decreased with parity and were lower at 150 to 280 DIM than in late lactation (Table 5).

The only diagnostic variable that could be evaluated at quarter level was the Acfu count. Quarters with CC23 showed significantly lower Acfu counts than quarters with $\mathrm{CC} 1$ or CC19 (Figure 2, bottom right; Table 6), mirroring the results obtained at the cow level using Ct values. In experimental studies conducted by Jensen (1982), bacterial shedding from quarters challenged with a human strain of $S$. agalactiae was lower than shedding from quarters challenged with a bovine strain. The difference in shedding was tentatively linked to a difference in transmissibility (Jensen, 1982). Strain-specific differences in transmissibility could lead to differences in within-cow or within-herd prevalence but, as stated above, our data do not lend statistical support for this hypothesis. Additional studies with a larger number of herds, multiple strains per herd, or experimental challenge models would be needed to provide a better understanding of strain-specific differences in pathogenesis of bovine mastitis and their relevance to transmission, diagnostics, and control of $S$. agalactiae mastitis.

\section{CONCLUSIONS}

Within-herd heterogeneity of $S$. agalactiae strains was observed based on PFGE and MLST, but a single strain and its derivatives dominated in each dairy herd, consistent with a contagious mode of transmission. In one herd with a large number of animals and a long history of infection, we observed considerable heterogeneity in PFGE patterns, which could have been misconstrued as an indication of environmental mastitis in the absence of MLST data and comparative PFGE data from other herds. In this preliminary study of the association between dominant CC of S. agalactiae and diagnostic characteristics, CC23 was associated with significantly higher $\mathrm{Ct}$ value at the cow level and lower approximate bacterial count (Acfu) at the quarter level

Table 5. Estimated coefficients of factors potentially associated with Ct-value for Streptococcus agalactiae in 46 cows from 6 dairy herds

\begin{tabular}{|c|c|c|c|c|}
\hline \multirow[b]{2}{*}{ Effect } & \multirow[b]{2}{*}{$\begin{array}{c}\text { Type } 3 \text { test of } \\
\text { fixed effect }(\operatorname{Pr}>\mathrm{F})\end{array}$} & \multicolumn{3}{|c|}{ Solution for fixed effects } \\
\hline & & Estimate & $\mathrm{SE}$ & $\begin{array}{c}P \text {-value } \\
(\operatorname{Pr}>|\mathrm{t}|)\end{array}$ \\
\hline Intercept & - & 36.59 & 1.88 & $<0.001$ \\
\hline Parity & 0.095 & -0.84 & 0.49 & 0.095 \\
\hline DIM & 0.017 & - & - & - \\
\hline$<150$ & - & $0.17^{\mathrm{a}}$ & 1.63 & 0.92 \\
\hline 150 to 280 & - & $-3.17^{\mathrm{b}}$ & 1.47 & 0.037 \\
\hline$>280$ & - & Reference $^{\text {ac }}$ & - & - \\
\hline Clonal complex (CC) & 0.118 & - & - & - \\
\hline CC 1 & - & -2.92 & 1.50 & 0.059 \\
\hline CC 19 & - & -3.00 & 15.4 & 0.059 \\
\hline CC 23 & - & Reference & - & - \\
\hline
\end{tabular}

${ }^{a-c}$ Different superscripts between levels of a factor indicate that they are significantly different. 
Table 6. Estimated coefficients of factors potentially associated with natural logarithm of approximate count (Acfu) for Streptococcus agalactiae in 71 infected quarters from 49 cows in 6 herds

\begin{tabular}{lcccc}
\hline & & \multicolumn{2}{c}{ Solution for fixed effects } \\
\cline { 3 - 5 } Effect & $\begin{array}{c}\text { Type } 3 \text { test of } \\
\text { fixed effect }(\operatorname{Pr}>\mathrm{F})\end{array}$ & Estimate & $\mathrm{SE}$ & $\begin{array}{c}P \text {-value } \\
(\operatorname{Pr}>|\mathrm{t}|)\end{array}$ \\
\hline Intercept & - & 3.16 & 0.46 & $<0.001$ \\
DIM & 0.0072 & - & - & - \\
$<150$ & - & $-0.32^{\mathrm{a}}$ & 0.40 & $<0.001$ \\
150 to 280 & - & $0.65^{\mathrm{b}}$ & 0.35 & $<0.001$ \\
$>280$ & 0.0118 & Reference $^{\mathrm{ac}}$ & - & - \\
Clonal complex (CC) & - & - & - & - \\
CC 1 & - & $1.23^{\mathrm{a}}$ & 0.40 & 0.0221 \\
CC 19 & - & Reference $^{\mathrm{b}}$ & - & 0.0030 \\
CC 23 & & - & - \\
\hline
\end{tabular}

${ }^{a-c}$ Different superscripts between levels of a factor indicate that they are significantly different.

compared with CC1 and CC19, indicating lower bacterial shedding by CC23-infected individuals. Observed median values for the number of infected quarters per cow and cow-level SCC were lower for cows infected with CC23 than for cows infected with CC1 or CC19, but those differences were not statistically significant. A considerable proportion of $S$. agalactiae-infected cows had SCC below the commonly used thresholds of 200,000 or 500,000 cells $/ \mathrm{mL}$ and might have gone undetected in an SCC-based screening and sampling program.

\section{ACKNOWLEDGMENTS}

We thank the participating farms for their hospitality as well as staff and students at the Moredun Research Institute (Penicuik, UK) and Streeklab Haarlem (Haarlem, the Netherlands) for help with molecular characterization of the isolates.

\section{REFERENCES}

Andersen, H. J., L. H. Pedersen, F. M. Aarestrup, and M. Chriél. 2003. Evaluation of the surveillance program of Streptococcus agalactiae in Danish dairy herds. J. Dairy Sci. 86:1233-1239.

Barkema, H. W., Y. H. Schukken, T. J. Lam, D. T. Galligan, M. L. Beiboer, and A. Brand. 1997. Estimation of interdependence among quarters of the bovine udder with subclinical mastitis and implications for analysis. J. Dairy Sci. 80:1592-1599.

Bliss, S. J., S. D. Manning, P. Tallman, C. J. Baker, M. D. Pearlman, C. F. Marrs, and B. Foxman. 2002. Group B Streptococcus colonization in male and nonpregnant female university students: A cross-sectional prevalence study. Clin. Infect. Dis. 34:184-190.

Boers, S. A., W. A. van der Reijden, and R. Jansen. 2012. Highthroughput multilocus sequence typing: Bringing molecular typing to the next level. PLoS ONE 7:e39630.

Delannoy, C. M., M. Crumlish, M. C. Fontaine, J. Pollock, G. Foster, M. P. Dagleish, J. F. Turnbull, and R. N. Zadoks. 2013. Human Streptococcus agalactiae strains in aquatic mammals and fish. BMC Microbiol. 13:41.

Dogan, B., Y. H. Schukken, C. Santisteban, and K. J. Boor. 2005. Distribution of serotypes and antimicrobial resistance genes among
Streptococcus agalactiae isolates from bovine and human hosts. J. Clin. Microbiol. 43:5899-5906.

Enright, M. C., and B. G. Spratt. 1999. Multilocus sequence typing. Trends Microbiol. 7:482-487.

Feil, E. J., B. C. Li, D. M. Aanensen, W. P. Hanage, and B. G. Spratt. 2004. eBURST: Inferring patterns of evolutionary descent among clusters of related bacterial genotypes from multilocus sequence typing data. J. Bacteriol. 186:1518-1530.

Ferguson, J. D., G. Azzaro, M. Gambina, and G. Licitra. 2007. Prevalence of mastitis pathogens in Ragusa, Sicily, from 2000 to 2006. J. Dairy Sci. 90:5798-5813.

Hansen, S. M., N. Uldbjerg, M. Kilian, and U. B. Sørensen. 2004 Dynamics of Streptococcus agalactiae colonization in women during and after pregnancy and in their infants. J. Clin. Microbiol. $42: 83-89$

Jensen, N. E. 1980a. Herd types of group-B streptococci. Their prevalence among herds in four Danish mastitis control areas and the relation of type to the spread within herds. Acta Vet. Scand. 21:633-639.

Jensen, N. E. 1980b. Distribution of serotypes of group-B streptococci in herds and cows within an area of Denmark. Acta Vet. Scand. 21:354-366.

Jensen, N. E. 1982. Experimental bovine group-B streptococcal mastitis induced by strains of human and bovine origin. Nord. Vet. Med. 34:441-450.

Jones, N., J. F. Bohnsack, S. Takahashi, K. A. Oliver, M. S. Chan, F. Kunst, P. Glaser, C. Rusniok, D. W. Crook, R. M. Harding, N. Bisharat, and B. G. Spratt. 2003. Multilocus sequence typing system for group B streptococcus. J. Clin. Microbiol. 41:2530-2536.

Kalmus, P., B. Aasmäe, A. Kärssin, T. Orro, and K. Kask. 2011. Udder pathogens and their resistance to antimicrobial agents in dairy cows in Estonia. Acta Vet. Scand. 53:4.

Katholm, J., T. W. Bennedsgaard, M. T. Koskinen, and E. Rattenborg. 2012. Quality of bulk tank milk samples from Danish dairy herds based on real-time polymerase chain reaction identification of mastitis pathogens. J. Dairy Sci. 95:5702-5708.

Keefe, G. P. 1997. Streptococcus agalactiae mastitis: A review. Can. Vet. J. 38:429-437.

Koskinen, M. T., J. Holopainen, S. Pyörälä, P. Bredbacka, A. Pitkälä, H. W. Barkema, R. Bexiga, J. Roberson, L. Sølverød, R. Piccinini, D. Kelton, H. Lehmusto, S. Niskala, and L. Salmikivi. 2009. Analytical specificity and sensitivity of a real-time PCR assay for identification of bovine mastitis pathogens. J. Dairy Sci. 92:952-959.

Mahmmod, Y. S., M. M. Mweu, S. S. Nielsen, J. Katholm, and I. C. Klaas. 2014. Effect of carryover and presampling procedures on the results of real-time PCR used for diagnosis of bovine intramammary infections with Streptococcus agalactiae at routine milk recordings. Prev. Vet. Med. 113:512-521.

Mahmmod, Y. S., N. Toft, J. Katholm, C. Grønbæk, and I. C. Klaas. 2013. Estimation of test characteristics of real-time PCR and bac- 
terial culture for diagnosis of subclinical intramammary infections with Streptococcus agalactiae in Danish dairy cattle in 2012 using latent class analysis. Prev. Vet. Med. 109:264-270.

Manning, S. D., A. C. Springman, E. Lehotzky, M. A. Lewis, T. S. Whittam, and H. D. Davies. 2009. Multilocus sequence types associated with neonatal group B streptococcal sepsis and meningitis in Canada. J. Clin. Microbiol. 47:1143-1148.

Manning, S. D., A. C. Springman, A. D. Million, N. R. Milton, S. E. McNamara, P. A. Somsel, P. Bartlett, and H. D. Davies. 2010. Association of Group B Streptococcus colonization and bovine exposure: a prospective cross-sectional cohort study. PLoS ONE 5:e8795.

National Mastitis Council. 1999. Laboratory Handbook on Bovine Mastitis. National Mastitis Council, Madison, WI.

Neave, F. K., F. H. Dodd, R. G. Kingwill, and D. R. Westgarth. 1969. Control of mastitis in the dairy herd by hygiene and management. J. Dairy Sci. 52:696-707.

Persson, K. M., B. Bjerre, L. Elfström, S. Polberger, and A. Forsgren 1986. Faecal carriage of group B streptococci. Eur. J. Clin. Microbiol. 5:156-159.

Phuektes, P., P. D. Mansell, and G. F. Browning. 2001. Multiplex polymerase chain reaction assay for simultaneous detection of Staphylococcus aureus and streptococcal causes of bovine mastitis. J. Dairy Sci. 84:1140-1148.

Piepers, S., L. De Meulemeester, A. de Kruif, G. Opsomer, H. W. Barkema, and S. De Vliegher. 2007. Prevalence and distribution of mastitis pathogens in subclinically infected dairy cows in Flanders, Belgium. J. Dairy Res. 74:478-483.

Reyher, K. K., S. Dufour, H. W. Barkema, L. Des Côteaux, T. J Devries, I. R. Dohoo, G. P. Keefe, J. P. Roy, and D. T. Scholl. 2011. The National Cohort of Dairy Farms - A data collection platform for mastitis research in Canada. J. Dairy Sci. 94:1616-1626.

Richards, V. P., P. Lang, P. D. Bitar, T. Lefébure, Y. H. Schukken, R. N. Zadoks, and M. J. Stanhope. 2011. Comparative genomics and the role of lateral gene transfer in the evolution of bovine adapted Streptococcus agalactiae. Infect. Genet. Evol. 11:1263-1275.

Runge, Ø., L. H. Pedersen, and J. Nielsen. 2004. Evaluation of the surveillance program for Streptococcus agalactiae (GBS) in Danish dairy herds. Danish Cattle Federation, Aarhus, Denmark.

Sampimon, O., H. W. Barkema, I. Berends, J. Sol, and T. Lam. 2009. Prevalence of intramammary infection in Dutch dairy herds. J. Dairy Res. 76:129-136.

Schukken, Y. H., D. J. Wilson, F. Welcome, L. Garrison-Tikofsky, and R. N. Gonzalez. 2003. Monitoring udder health and milk quality using somatic cell counts. Vet. Res. 34:579-596.

Shome, B. R., M. Bhuvana, S. D. Mitra, N. Krithiga, R. Shome, D. Velu, A. Banerjee, S. B. Barbuddhe, K. Prabhudas, and H. Rah- man. 2012. Molecular characterization of Streptococcus agalactiae and Streptococcus uberis isolates from bovine milk. Trop. Anim. Health Prod. 44:1981-1992.

Smith, T. H., L. K. Fox, and J. R. Middleton. 1998. Outbreak of mastitis caused by one strain of Staphylococcus aureus in a closed dairy herd. J. Am. Vet. Med. Assoc. 212:553-556.

Sørensen, U. B., K. Poulsen, C. Ghezzo, I. Margarit, and M. Kilian. 2010. Emergence and global dissemination of host-specific Streptococcus agalactiae clones. MBio 1:e00178-10.

Springman, A. C., D. W. Lacher, E. A. Waymire, S. L. Wengert, P. Singh, R. N. Zadoks, H. D. Davies, and S. D. Manning. 2014. Pilus distribution among lineages of group b streptococcus: An evolutionary and clinical perspective. BMC Microbiol. 14:159.

Stableforth, A. W. 1950. Bovine mastitis with particular regard to eradication of Streptococcus agalactiae. Vet. Rec. 62:219-224.

Struelens, M. J., Y. De Gheldre, and A. Deplano. 1998. Comparative and library epidemiological typing systems: Outbreak investigations versus surveillance systems. Infect. Control Hosp. Epidemiol. 19:565-569.

Tenhagen, B. A. G. Köster, J. Wallmann, and W. Heuwieser. 2006 Prevalence of mastitis pathogens and their resistance against antimicrobial agents in dairy cows in Brandenburg, Germany. J. Dairy Sci. 89:2542-2551.

Tenover, F. C., R. D. Arbeit, R. V. Goering, P. A. Mickelsen, B. E. Murray, D. H. Persing, and B. Swaminathan. 1995. Interpreting chromosomal DNA restriction patterns produced by pulsed-field gel electrophoresis: criteria for bacterial strain typing. J. Clin. Microbiol. 33:2233-2239.

Thieme, V. D., and S. Haasmann, 1978. Variation in Sc. agalactiae excretion-Consequences for diagnosis and control of infectious mastitis. Monatsh. Veterinarmed. 33:298-304. [In German]

van der Mee-Marquet, N., L. Fourny, L. Arnault, A. S. Domelier, M. Salloum, M. F. Lartigue, and R. Quentin. 2008. Molecular characterization of human-colonizing Streptococcus agalactiae strains isolated from throat, skin, anal margin, and genital body sites. J. Clin. Microbiol. 46:2906-2911.

Yang, Y., Y. Liu, Y. Ding, L. Yi, Z. Ma, H. Fan, and C. Lu. 2013. Molecular characterization of Streptococcus agalactiae isolated from bovine mastitis in Eastern China. PLoS ONE 8:e67755.

Zadoks, R. N., B. E. Gillespie, H. W. Barkema, O. C. Sampimon, S. P. Oliver, and Y. H. Schukken. 2003. Clinical, epidemiological and molecular characteristics of Streptococcus uberis infections in dairy herds. Epidemiol. Infect. 130:335-349.

Zadoks, R. N., J. R. Middleton, S. McDougall, J. Katholm, and Y. H. Schukken. 2011. Molecular epidemiology of mastitis pathogens of dairy cattle and comparative relevance to humans. J. Mammary Gland Biol. Neoplasia 16:357-372. 